International Journal of Engineering \& Technology, $7(2.19)(2018) 21-25$
International Journal of Engineering \& Technology
SPC
Website: www.sciencepubco.com/index.php/IJET
Research paper

\title{
Emotion Recognition Based on Generalized Gamma Distribution
}

\author{
Sujeeth .T ${ }^{1 *}$, Y.Srinivas ${ }^{2}$, Nagesh Vadaparthi ${ }^{3}$ \\ ${ }^{1}$ Asst Professor, Dept CSE, Siddartha Educational Academy Group of Institutions, Tirupati, \\ Research Scholar, Department of CSE, GIT,GITAM \\ ${ }^{2}$ Professor, Department of IT, GIT, GITAM \\ ${ }^{3}$ Professor, Department of IT, MVGR College of Engineering, Vizianagaram
}

\begin{abstract}
It is highly difficult to identify the emotions of a person. Literature accessible to recognize the emotions in case of immobilized personnel is limited to the outcome obtainable from the machines only. In this process, brain computer communication is utilized using neuro-scan machines like Encephalography (EEG), to recognize the feeling of immobilized persons. It uses the physiological signals accessible from EEG data extracted from the brain signals of immobilized personnel and tries to find out the emotions, but these results vary from machine to machine, and there exists no consistency by which one can identify the thoughts of the brain diseased personnel precisely. In this manuscript a novel technique is projected by using Generalized Gamma Mixture models (GGMM). The advantage of considering GGMM is its ability of extracting the emotions closely even in a noisy environment. The outcomes of the proposed method exceed the accuracy rates of conventional systems.
\end{abstract}

Keywords: Emotion recognition, Gamma Mixture Model, Encephalography, immobilized persons, Recognition rates

\section{Introduction}

In the recent years, the advancement in the technology, the human computer interface has become an important aspect when, the intention of the user is analyzed and based on his intention the computer performs the action. Hence, there is a huge need to focus on the human intentions and the computer's response to it. The intentions are again dependent on emotions of a person. In fact, emotions play a vital role on the cognitive capabilities and the activities that depend on cognition of a human [1]. The emotions of humans through facial expressions or through voice can easily be detected and identified [2]. But it is very difficult to analyze the emotions of the patients who are dumb or even in small children. Hence EEG is the only source of input for the doctors to identify the emotions of children or patients suffering with expression problem.

According to the Psychologists every human emotion can be classified into different facial expressions (angry, sad, disgust, happy, fearful and surprise). These expressions are mostly constant and are not independent of the aging factors. Most of the automatic human emotion identification systems are not concrete. Since emotions express the intuitive state of mind of an individual, the apparent expression can be willfully controlled as per the situations. For instance, a person with anger exhibits the same before his wife or children and the same anger is projected before his boss in a different way. Therefore, the outward actions may not be exactly concrete. Therefore, to have a exact identification of the human expressions, EEG signals can be more reliant [3] .EEG signal alter in tune with the emotional state of the individual, which cannot be tampered or articulated, and thus become the true indicators of the individual reactions and reflections at a particular instance.

The EEG signals are generated basing on the human emotions and expressions are the central nervous system and the automatic nervous system. The central nervous system consists of spinal card and the brain. To analyze the signals and measure the emotions, there are various methods viz., Galvanic skin response (GSR), Electromyography (EMG), Heart rate(HR), Respiration Rate (RR) and Electro Encephalography(EEG) etc[4]. However, EEG is proved to be the direct mode of studying the brain data [5][6]. Though EEG has less spatial resolution, it provides high time resolution. The acquiring EEG data is fast and inexpensive. Also, the EEG device is portable, less costly, easy to use and its usage make the researchers consider EEG widely and can be applied in the areas of entertainment, e-learning, e-health care etc[7],[8],[9],[10] . These signals differ in shape and possess long tails, therefore to interpret these signals and classify the emotions based on non-parameter models will be a challenging task $[11],[12,[13],[14]$ and parametric models are therefore assumed to be more suitable for these purposes [8][9],[14],[15]. However, the parametric models based approach is considered to analyze the human emotions in this article.

The paper is articulated as follows, Section 2 highlights about EEG, and section 3 gives a brief review on research work so far done for emotion recognition using EEG. Section 4 presents the gap observed in the research area based on the previous section. Section 5 highlights the proposed model based on Generalized Gamma distribution. Section -6 presents the details on experimentation and results obtained. Section - 7 presents the methodology and the conclusions are presented in section 8 . 


\section{Electroencephalography (EEG)}

Modern high-resolution Electroencephalography (EEG) and magneto-encephalography (MEG) techniques allow investigating noninvasively in humans spatially selective effects in electric and magnetic brain activity. When restricted to the channel (electrode or sensor) level, the spatial analysis of the EEG and MEG sources can only provide limited information about the cortical regions involved in the generation and the perturbation of these effects. Both wideband signals like event-related potentials (ERP) and event-related fields (ERF) and narrow-band (i.e. rhythmic synchronization) effects like event-related spectral perturbation (ERSP) and phase coherence (PC) can be originated from one or multiple cortical sites, thereby making the characterization of their cortical distribution potentially important for strengthening notions of functional specialization and building new augmented spatio-temporal models of functional integration.

\subsection{Emotion Categories}

The human emotions are basically classified into three types: Motivational (Thirst, Hunger, Pain, Mood), Basic (Happy, Sad, Fear, Disgust, Anger, and Surprise) and Self Conscious or Social (Shame, Embarrassment, Pride, and Guilt). Pattern recognition efforts aimed at finding physiological correlates, focusing on $t$ tests or Analysis of Variance (ANOVA) comparisons and combining data over many subjects, where each was measured for a relatively small amount of time.

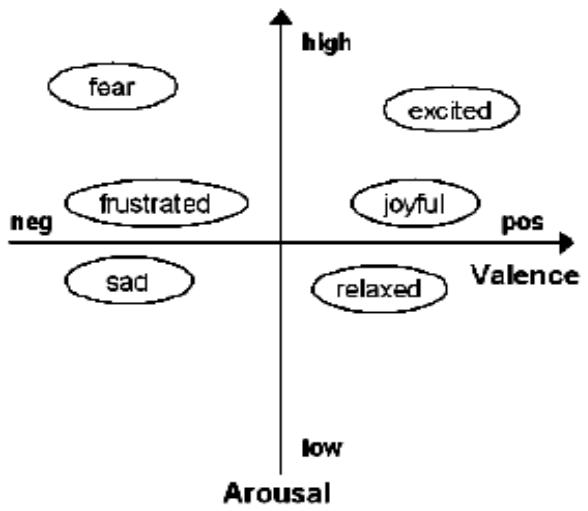

Fig.1(a): Discussion Model for Emotion
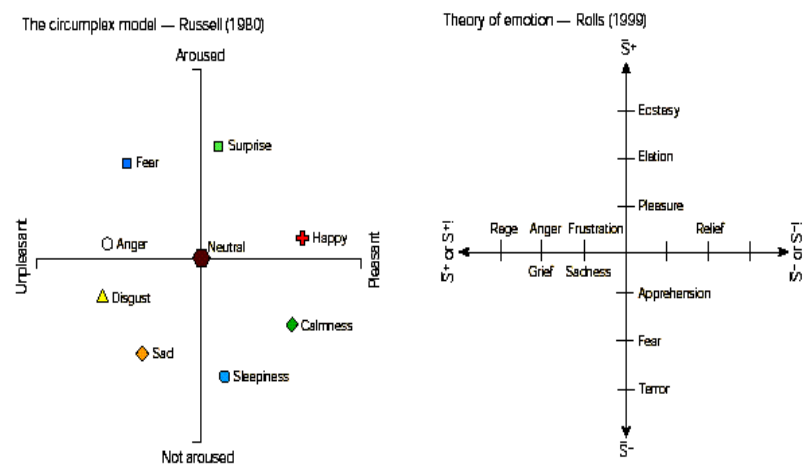

Fig.1(b): Discussion Model for Emotion

One of the researcher Lang P.J[16], claimed that emotions can be characterized in terms of judged valence (Pleasant or unpleasant) and arousal (calm or aroused). The above Fig 1 (a) and Fig 1(b), shows the region of relation between arousal with valence. The relation between physiological signals and arousal/valence is established due to the activation of the automatic nervous system when emotions are elicited

\subsection{Electrical Brain Activity}

To monitor electrical brain activity, electroencephalography is used. The electroencephalogram (EEG) provides information about the electrical activity of the human brain which results from post synaptic potentials of the cortical neurons. Compared to other methods for monitoring brain activity such as functional Magnetic Resonance Imaging (fMRI) or Proton Emission Tomography (PET), EEG has a high temporal resolution. However, as the origin of the signal cannot be localized precisely, the special resolution of the EEG is worse than the special resolution of other methods.

There are two different types of EEG signals:

1. The spontaneous EEG measures the continuous activity which has amplitudes range 10 and $100 \mathrm{uV}$ [17]. According to international conventions the frequencies that can be observed are subdivided into different frequency bands: $\delta(1-4 \mathrm{~Hz}), \theta(4-7 \mathrm{~Hz}), \alpha(8-13 \mathrm{~Hz}), \beta(13-30$ $\mathrm{Hz})$ and $\gamma(\geq 30 \mathrm{~Hz})$ bands[18]

2. Evoked potentials occur as a response while stimuli (e.g. a visual ash of light or an auditory click) are presented. The duration of this event is usually less than $500 \mathrm{~ms}$. To measure EEG, electrodes are placed on the surface of the skull. Each electrode measures the electric potential of the surrounding head area. The signal can be conducted unipolar or bipolar. When using the unipolar method a so-called active electrode is placed on the area of interest and a reference electrode is placed on a relatively inactive area like the earlobe. In contrast, using the bipolar recording technique requires two active electrodes placed over the cortical areas of interest.
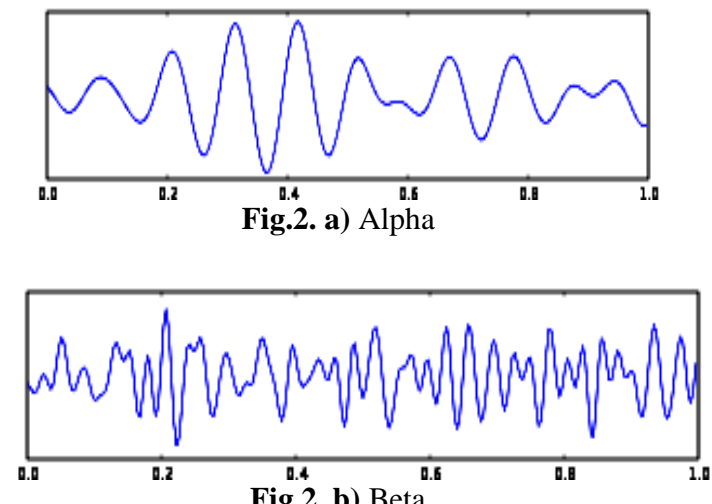

Fig.2. b) Beta
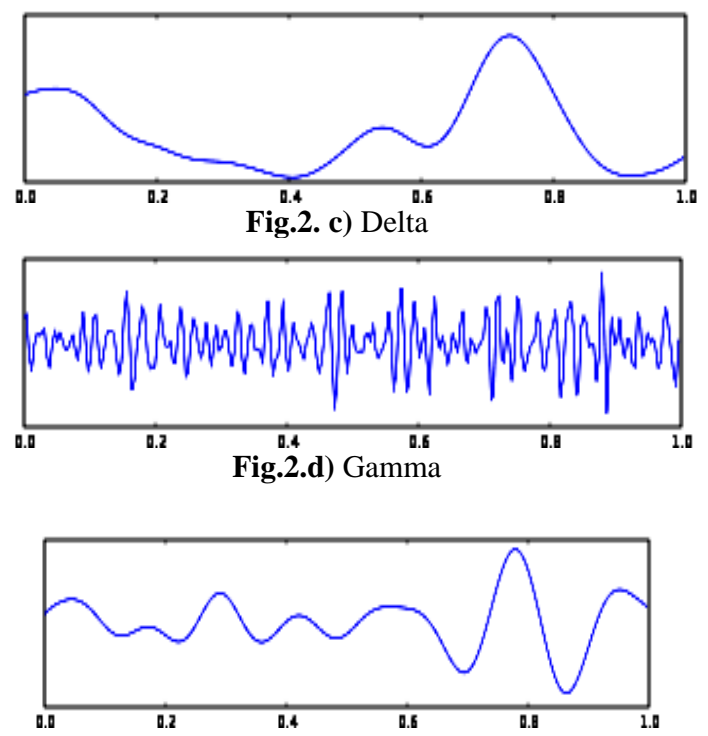


\section{Fig.2 e) Theta}

Either the difference or the sum of the electric potentials between the two electrodes is recorded. One major advantage of bipolar recordings can be seen in the fact that no inactive reference area has to be selected (this can be quite problematic as there's no place which is completely inactive). However, the main disadvantage of bipolar recordings is that a combined activity of two locations is produced what makes it useless for clinical and research purposes where data of symmetrically placed electrodes or activity of special brain areas has to be compared.

\section{Related Works on Emotion Recognition Us- ing Eeg}

Power spectral compactness (PSC) and brain irregularities (BAY) acquired from the EEG signals are considered by [19] together with Support vector machine classifier for the identification of the emotions. Their works concluded that they have attained $93.31 \%$ of accuracy rate in case of PSC and $85.39 \%$ with BAY. DRBM based recognition system was proposed by [20] in which the system was developed by considering the hidden relationship among the user profiles, EEG and emotion labels. Statistically Weighed Autoregressive Modelling of Electroencephalogram (EEG) signals was presented by [21] in order to acquire the emotions and the authors claims about an accuracy of $94.097 \%$. In [22] have projected a paper which identifies the EEG brain signals together with speeches articulated in different languages to make out human emotions. Methods used are Emotion tracking and Higher Order crossings and Empirical Mode Decomposition. Feature Extraction is done based on Independent Component Analysis (ICA), Mel frequency cepstral coefficient (MFCC), KNN method, and wavelet.

Authors of [6] have projected a paper in which the authors have concerted to distinguish the emotions based on EEG feature extraction. Results claim that their model could achieve about $62.3 \%$ mean successful rate, for the mixture channel case, and $83.33 \%$ mean classification rate when SVM classifier is considered. [22] in their paper identified the human emotions with the help of wavelet transform using EEG signals. Here they are considering five different emotions namely natural, happy, fear, disgust, and surprise. They have considered an EEG with 64 electrodes to collect EEG signals. Pre-processing is done with the help of SL filter. They are classifying the emotions with the help of DTW. KNN and LDA are utilized for performance evaluation and emotional state classification. The accuracy rate is $83.26 \%$. [23] proposed a paper focuses on classification of EEG have shown a relationship on emotions with the help of features extracted GM of EEG spectrogram. Fractional dimension of EEG signal is compared with three feature extraction methods, for features extraction they are using KNN and SVM

They have carried out their works on four different emotions; optimistic, pessimistic tranquil and thrilled. To extract the features Gaussian mixture model is considered. [24] has considered a real time system to make out the emotions using EEG and they have carried out the sensitivity analysis in presence of noise. In their projected work, they have identified the features by considering the wavelet transforms and for the classification, different classifiers were considered. [22] presented an article by considering physically immobilized persons. In this article, the authors have tried to develop a model for establishing a communication medium to interact with the immobilized people. The authors have tried to identify the emotions like happy disgust, fear and surprise. With the help of wavelet functions they extracted the statistical features of human brain signals (EEG). [25] estimated a proficient gaining procedure for congregation the EEG signals. For the preprocessing AMR method was used. The experimentation was carried out on six healthy people using 63 biosensors. Discrete Wavelet Transforms are considered for the extraction of EEG parameters and the classification of the emotions are carried out by using Fuzzy C-Means (FCM).

\section{GAP Observed}

In order to obtain the emotions from the immobilized persons or to identify the exact emotions from the individuals, EEG is mostly preferred in the literature. To extract these signals, EEG electrodes are placed on the brain scalp. The emotion signals that are exhibited from the human brain are inferred from the EEG using the limbic system. Ranges of regions in the brain, that contribute in evaluating the emotions from the human brain include, 1. Left Temporal areas: sadness. 2. Right Prefrontal Cortex area: sadness, happiness and disgust. 3. Bilateral Temporal activation: fear. Apart from the above, all emotions share the areas viz., prefrontal cortex, cingulated gyrus and temporal cortex [13],[14],[15]. In order to extract the EEG signals, the individuals are to be trained by explaining them about the system and then position the electrodes. The extraction of the signals needs to be carried out in presence of a trained technician. In most of the cases, Fp1, FpZ, Fp2, F7, F3, F2, F4 and F8 electrodes are considered to interpret. The emotions exhibit different shapes of signals and to understand these signals different techniques like bio-potential signal processing techniques, arousal-valence plane based systems, frequency domain techniques and Gaussian model based techniques are mostly projected in the literature [22],[23],[25]. However, these techniques fail to model the system having elongated tails and in the noisy environments. Therefore, to overcome these limitations, in this article, Generalized Gamma Distribution is considered. Generalized Gamma Distribution includes several other distributions such as Weibull distribution, Gamma distribution, Log-normal distribution, Chi-square distribution and Exponential distribution as particular cases. The main advantage of using Generalized Gamma Distribution is that, it can sensitize the emotions from the signals acquired even in the presence of minimum variance more effectively.

\section{Generalized Gama Mixture Model}

The generalized gamma distribution is a continuous probability distribution with three parameters $a>0, d>0$, and $p>0$. It is a generalization of the two-parameter gamma distribution. As many distributions are commonly used for parametric models in survival analysis (such as the Exponential distribution, the Weibull distribution and the Gamma distribution) are special cases of the generalized gamma.

Generalized Gamma Distribution is projected in this article for the categorization of the emotions based on the EEG signals. In general, in extracting the signals from the brain, the underlying emotions exhibiting from each of these signals are to be interpreted based on the intensity levels. In order to interpret these variation in signals, tail distributions will be more appropriate and to classify the signals having minimum variations, the best suited distribution is Generalized Gamma Distribution.

The shorthand $X \sim$ generalized gamma $(\alpha, \beta, \gamma)$ is used to indicate that the random variable $X$ has the generalized gamma distribution with real positive parameters $\alpha, \beta$, and $\gamma$. A generalized gamma random variable $X$ with scale parameter $\alpha$, and shape parameters $\beta$, and $\gamma$ has probability density function

$$
f(x)=\frac{\gamma x^{\gamma \beta-1} e^{-(x / \alpha)^{\gamma}}}{\alpha^{\gamma \beta} \Gamma(\beta)} \quad x>0 .
$$


The probability density function with three different parameter combinations is illustrated below

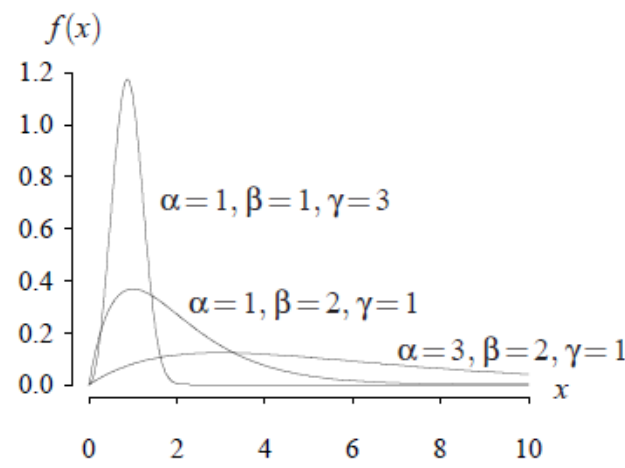

Fig.3. PDF with 3 parameters

\section{Methodology}

The EEG data is acquired from the equipment with the help of 64 channel cap wearing by the subject. The subject has to be initially trained regarding the process of acquiring the data corresponding to different emotions and the care that has to be taken while acquiring the data as the process/equipment is sensitive to movements. Though the 64 electrode eliminate the problem of less temporal sensitivity, the signals acquired from only few electrodes are considered viz., fp1, fpZ, fp2, f7, f3, f2, f4 \& f8 [10],[11]. The obtained frequencies are trained using the Generalized Gamma distribution.

Algorithm of the proposed model for identifying the emotion is as follows:

Step - 1: Train the emotional dataset

Step - 2: Finding MU \& SIGMA values of the training dataset

Step - 3: Calculate the Probability Density function of all emotional datasets

Step - 4: Extract the EEG Brain signals (Test database)

Step - 5: Find the MU \& SIGMA values of the Test data

Step - 6: Calculate the PDFs of the Test data.

Step - 7: Compare the Test data with the Train database and identify which emotion data does the EEG matches with the existing dataset.

Step - 8: Displays the Emotion

\section{Results and Discussions}

For experimentation, eight electrodes configuration, only the BLUE electrodes were used. The electrode is placed with different positions such as 10-10 electrodes system, 10-20 electrode systems, 64 electrode systems according to the application. Dataset used for testing is SEED dataset and DEAP dataset. In this experimentation process we have considered dataset related 5 subjects. However, the performance improvement of the algorithm shall increase as the data size increases.

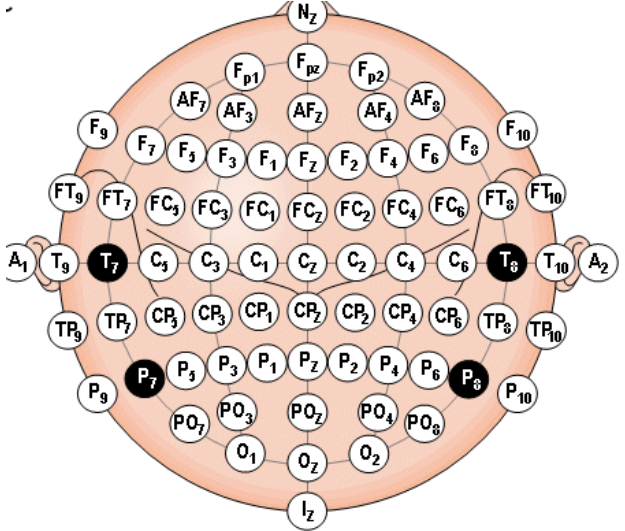

Fig.4. Placement of 64 electrodes on head

Table 1: Trial IDs and their emotion states

\begin{tabular}{|c|c|}
\hline Trial IDs & Induced Emotion \\
\hline $1,6,9,10,14$ & Positive \\
\hline $2,5,8,11,13$ & Neutral \\
\hline $3,4,7,12,15$ & Negative \\
\hline
\end{tabular}

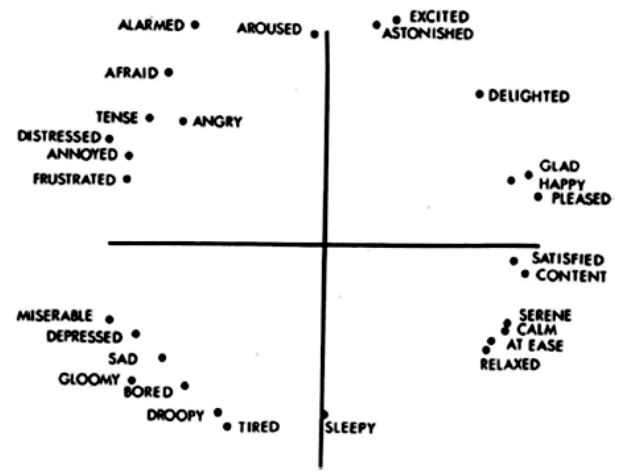

Fig.5: Russel's Circumplex Model of Affect [26]

The experimentation is done on 2 subjects and the dataset using MATLAB on dual core processor, 4GB RAM, and HDD.

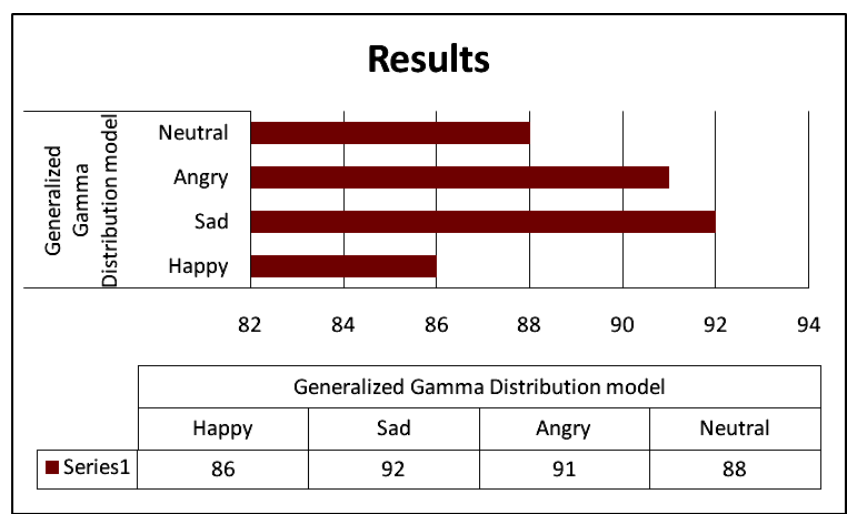

Fig.6: Graph showing \% Accuracy

Table 2: Emotion recognition accuracy rates

\begin{tabular}{|c|c|c|c|c|}
\hline \multirow{2}{*}{ Name of the classifier } & \multicolumn{4}{|c|}{$\begin{array}{c}\text { Generalized Gamma Distribution } \\
\text { model }\end{array}$} \\
\hline $\begin{array}{c}\text { Data acquisition meth- } \\
\text { od: Electroencephalo- } \\
\text { graph }\end{array}$ & Happy & Sad & Angry & Neutral \\
\cline { 2 - 5 } & 86 & 92 & 91 & 88 \\
\hline
\end{tabular}




\section{Conclusion}

Thus the experimental results clearly highlight the effectiveness of using Generalized Gamma mixture model for identifying the emotions using EEG. The results of the proposed system surpassed the results of conventional models. The proposed method further can be enhanced by adding the voice signal to correlate and accurately identify emotion.

\section{References}

[1] Ning Zhuang et al,"Emotion Recognition from EEG Signals Using Multidimensional Information in EMD Domain", BioMed Research International, Vol.2017, (2017), pp.01-09, available online: https://doi.org/10.1155/2017/8317357

[2] Gunes $\mathrm{H}$ et al,"Emotion representation, analysis and synthesis in continuous space:A Survey", IEEE FG, 2011 pp:827-834.

[3] S. J. Young, G. Evermann, M. J. F. Gales, T. Hain, D. Kershaw, G. Moore, J. Odell, D. Ollason, D. Povey, V. Valtchev, and P. C.' Woodland, The HTK Book, version 3.4, 2006.

[4] Soraia M.Alarcao and Manuel J F, "Emotions Recognition Using EEG Signals:A Survey", IEEE Transactions on Affective Computing, pp:01-20, 2017, DOI:10.1109/TAFFC.2017.2714671 .

[5] Priyanka A. Abhang ,'Introduction to EEG- and Speech-Based Emotion Recognition" Academic Press, 2016

[6] Panagiotis C, "EEG based emotion recognition, using Advanced signal processing techniques," Wiley publications, 2010

[7] D. Reynolds, T. Quatieri, and R. Dunn, "Speaker verication using adapted Gaussian Mixture Model," Digital Signal Processing, July 2000

[8] N. Thapliyal and G. Amoli, "Speech based emotion recognition with Gaussian Mixture Model," International Journal of Advanced Research in Computer Engineering \& Technology, 2012.

[9] J. Sohn, N. Kim, and W. Sung, "A statistical model based voice activity detector," IEEE Signal Processing Letters, vol. 6, pp. 13, 1999.

[10] Ivan Tashev, Andrew Lovitt, and Alex Acero, "Dual stage probabilistic voice activity detector," in NOISE-CON 2010 and 159th Meeting of the Acoustical Society of America, 2010.

[11] Srinivas Yarramalle, J.Sirisha Devi, N.Murali Krishna, “A Novel Approach for Effective Emotion Recognition Using Double Truncated Gaussian Mixture Model and EEG', I.J. Intelligent Systems and Applications, 2017,vol 6, pp 33-42

[12] J. Sirisha Devi, Y. Srinivas, "Automatic Speech Emotion and Speaker Verification based on Hybrid GMM and FFBNN", International Journal on Computational Sciences \& Applications (IJCSA) Vol.4, No.1, February 2014, ISSN: 2200-0011Prasad Reddy P.V.G.D, A. Prasad, Y. Srinivas, P. Brahmaiah, "Gender Based Emotion Recognition System for Telugu Rural Dialects Using Hidden Markov Models" Journal of Computing, Vol. 2, No. 6, , pp 23-30,June 2010

[13] J. SirishaDevi, Dr. Srinivas Yarramalle, Siva Prasad Nandyala "Speaker Emotion Recognition Based on Speech Features and Classification Techniques" I.J. Image, Graphics and Signal Processing, 2014, No:7, pp: 61-77, June 2014.

[14] K. Takahashi, "Remarks on Emotion Recognition from BioPotential Signals," in Proceedings of the 2nd International Conference on Autonomous Robots and Agents, 2004, pp. 186-191.

[15] X. Wang, D. Nie, and B. Lu, "EEG-based emotion recognition using frequency domain features and support vector machines," Neural Information Processing, pp. 734-743, 2011.

[16] Lang P.J., Bradley, "Emotion, attention and the startle reflex",Psychological Review, Vol.97, pp:377-398, 1990

[17] Teplan M., "Fundamentals of EEG measurement",Measurement Science Review, pp. 01-11, 2002.

[18] Niedermeyer and F.Da Silva, "Electroencephalography: Basic Principles, Clinical Applications and Related Fields", Lippincott Willianms \& Wilkins, 2005

[19] Shuang Liu et al, "EEG-based Emotion Recognition Using Nonlinear Feature", IEEE 8th International Conference on Awareness Science and Technology (iCAST 2017), pp. 55-59, DOI: 10.1109@ICAwST.2017.8256518.

[20] Kevin C. Tseng, Bor-Shyh Lin, "Emotion Recognition of EEG Underlying Favorite Music by Support Vector machine”, International Conference Orange Technologies (ICOT) 2017

[21] Aravind E Vijayan, Deepak Sen, Sudheer A.P "EEGbased Emotion Recognition using Statistical measures and Auto-regressive modeling", 2015 IEEE International Conference on Computational Intelligence \& Communication Technology, 2015 IEEE.

[22] M.Murugappan "Human Emotion Recognition Through Short Time Electroencephalogram (EEG) Signals using Fast Fourier Transform", 2010 IEEE 9th International Colloquium on Signal Processing and its Applications

[23] Reza Khosrowabadi," EEG based emotion recognition using MFCC and MLP", Proceedings of ISCA 25th International Conference on Computers and Their Applications, CATA 2010 March 24-26, 2010,

[24] Ali S. AlMejrad (2010), “A Novel Technique for Extraction Foetal Electrocardiogram using Adaptive Filtering and Simple Genetic Algorithm", Current Research in Biostatistics, Vol. 1, No. 2 , pp.75-81, DOI : 10.3844/amjbsp.2010.75.81.

[25] Murugappan Murugappan, Nagarajan Ramachandran, Yaacob Sazali,"Classification of human emotion from EEG using discrete wavelet transform", Pattern Recog.2009 [6]

[26] Russell JA, "A circumplex model of affect", J Personal SocPsychol 39(6):1161-1178, 1980. 